\title{
Méthodologie d'instrumentation d'un ouvrage constitué de parois moulées à contreforts : le $\mathrm{B} 600$
}

\author{
L. Delattre ${ }^{1}$, R.Boutin ${ }^{2}$, M. Pioline ${ }^{3}$, G. Vinceslas ${ }^{3}$
}

\section{Résumé}

Le B600 est un moyen d'essai de la carène des navires. L'élément principal de l'ouvrage est une cuve de $545 \mathrm{~m}$ de long, $15 \mathrm{~m}$ de large et de $8 \mathrm{~m}$ de profondeur, réalisée en excavation entre des parois moulées à contreforts autostables. Les méthodes de calcul de ce type d'ouvrages de soutènement soulèvent encore des interrogations, aussi une instrumentation de la structure (tubes inclinométriques, nivelles, plots pour mesure de déplacement au fil Invar, plots topographiques) a été mise en place afin de suivre la cinématique des éléments de paroi moulée et du terrain associé. L'étude a également porté sur le niveau de sollicitation des éléments de paroi moulée au moyen d'extensomètres à jauges et à cordes vibrantes. Cette instrumentation a permis de vérifier que le comportement de l'ouvrage était proche de celui attendu en ce qui concerne les déplacements d'ensemble et que la déformation des éléments de paroi moulée restait faible. Cette instrumentation apporte également des éléments de comparaison avec les structures mieux connues que sont les soutènements plans et les murs poids.

New tank for testing French Navy ships models is a $545 \mathrm{~m}$ long, $15 \mathrm{~m}$ wide and $8 \mathrm{~m}$ deep canal called B600. It is composed of two cantilever diaphragm walls between which ground has been excavated. Cantilever diaphragm walls are buttressed walls ( $T$ section), for which design remains questionable. As a consequence, their behaviour during excavation was monitored, in order to ensure the safety of excavation work and to get some new informations on the behaviour of such structures. Monitoring program included inclinometers, surveying plots of ground and wall movements, gages and vibrating wire extensometers set in the concrete. It proved correct a behaviour of the structure and provided new informations on the behaviour of such buttressed cantilever walls.

\section{Introduction}

Les parois moulées à contreforts constituent une technique de soutènement qui, sans être de premier plan, est néanmoins d'un emploi courant (Delahaye et al., 1998). Elles peuvent en particulier être utilisées pour la réalisation de soutènements autostables de grande hauteur (Lavisse, 1993; Parkinson et Guillaud, 1994), les contreforts participant, d'une part, à l'obtention d'écrans de

\footnotetext{
${ }^{1}$ LCPC, 58, boulevard Lefebvre, 75732 Paris cedex 15 ,

${ }^{2}$ STTIM, 15 rue de Laborde, 00309 Armées,

${ }^{3}$ CETE Normandie-Centre, Chemin de la Poudrière - BP 245, 76121 Le Grand-Quevilly cedex.
} 


\section{VII ${ }^{\text {èmes }}$ Journées Nationales Génie Civil - Génie Côtier, Anglet, France, 15-17 Mai 2002}

forte inertie et donc à la limitation de leur déformation propre et, d'autre part, à l'ancrage de la paroi dans le terrain.

Les schémas d'interaction sol-structure qui sont mis en jeu par ce type d'ouvrage ne sont toutefois qu'imparfaitement maîtrisés. Ainsi, si l'espacement et la longueur des contreforts gouvernent de façon évidente le comportement de l'ouvrage, il n'existe actuellement pas de modèle de calcul de ces ouvrages communément admis et permettant de tenir compte de l'ensemble des valeurs susceptibles d'être prises par ces paramètres. Les modèles de calcul de l'ouvrage poids (contreforts suffisamment rapprochés pour que le sol emprisonné entre les contreforts puisse être considéré comme solidaire de la paroi) et de l'écran plan (les contreforts n'interviennent plus que dans l'inertie de l'écran) ne constituent que deux approches encadrant le problème à la limite. Du point de vue du dimensionnement de la structure, la situation n'est pas plus satisfaisante, la distribution des efforts dans les contreforts et dans le voile, en termes d'orientation, de sens et d'intensité, n'étant pas connue de façon précise.

Le choix de la technique de la paroi moulée à contreforts pour la réalisation des bajoyers du B600, en soulevant ces questions d'interaction sol-structure, a conduit le service d'infrastructures de la Marine Nationale à accompagner la construction de l'ouvrage de l'expérimentation d'une section de celui-ci. L'expérimentation avait pour objet, d'une part, de s'assurer que le comportement de l'ouvrage était bien le comportement attendu et, d'autre part, de contribuer à l'amélioration des méthodes de calcul en apportant des nouveaux éléments de compréhension de son fonctionnement. Après une présentation des caractéristiques de l'ouvrage, l'article présente l'instrumentation réalisée et les principaux résultats obtenus.

\section{Le site et l'ouvrage}

Le Bassin de traction B600 est l'un des nouveaux moyens d'essais du site du Bassin d'essais des carènes à Val-de-Reuil (Cota, 1996; Figure 1). Les essais sont réalisés avec des maquettes à échelle réduite afin d'étudier expérimentalement les performances d'engins se déplaçant dans un fluide. C'est plus particulièrement les phénomènes à l'interface air/eau qui sont étudiés. Les caractéristiques dimensionnelles du B600, $545 \mathrm{~m}$ de long, avec une veine d'eau ayant une section de $15 \mathrm{~m} \times 7 \mathrm{~m}$, découlent de l'examen des paramètres de similitude permettant de passer de la nature au modèle réduit. Les dimensions du bassin se justifient pour la réalisation des essais de remorquage pour l'étude de la traînée, des essais d'autopropulsion pour l'étude de la puissance de la machine, des essais de tenue à la mer pour l'évaluation du comportement du navire sur la houle et des essais de manœuvrabilité pour mesurer les efforts supportés par la carène lors de manœuvres (Boloré et Cordier, 1999). 

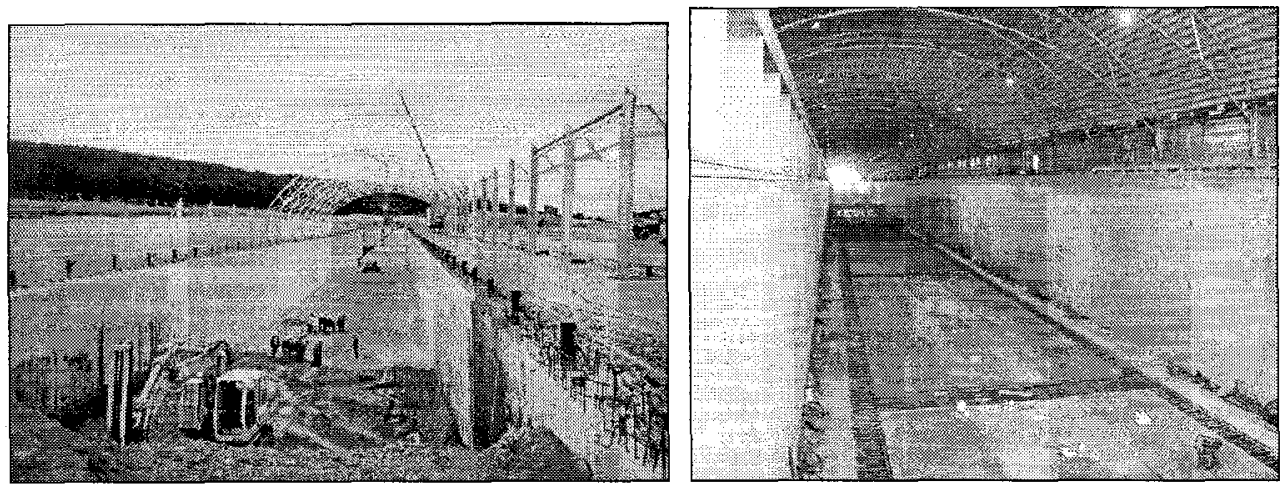

Figure 1 - Vues de la cuve du B600 en cours de construction.

Figure 1-B600 tank under construction.

L'ouvrage est implanté sur le versant ouest de la vallée de l'Eure, non loin de son confluent avec la Seine (secteur de la boucle de Poses), à une altitude moyenne de 40 NGF. L'emprise du bassin, orienté nord-sud, suit sensiblement les courbes de niveau, l'altitude variant seulement entre 38,5 et 42,5 NGF sur le linéaire total du bassin. Sur les deux-tiers nord de l'ouvrage, secteur où se situe la zone instrumentée, les investigations géotechniques ont montré une succession assez régulière de couches (Figure 2). Des limons sont rencontrés en moyenne depuis la surface du sol jusqu'à la cote 32 NGF. Ils se rattachent à deux catégories, des limons marrons, argileux, avec cailloutis, constituant des dépôts de solifluxion, rencontrés en surface jusqu'à une épaisseur de $1,50 \mathrm{~m}$, et des limons sableux d'origine éolienne. Des alluvions sablo-graveleuses sous-jacentes sont identifiées, à l'altitude du site, compte-tenu du contexte géologique, comme des lambeaux de terrasses alluviales de l'Eure ou de la Seine. Les matériaux constitutifs sont respectivement, soit des graves creuses limoneuses, soit des graves sableuses. Le substratum crayeux, dont le niveau d'érosion se situe le plus souvent entre 25 et 30 NGF, se rattache à la base de l'étage Sénonien, sous-étage Coniacien, et est constitué d'une craie dure avec bancs de silex. Cette craie a été le siège de nombreux phénomènes karstiques qui se traduisent par la présence de cavités franches ou de poches remplies de matériau amené depuis les couches supérieures par les eaux d'infiltration.

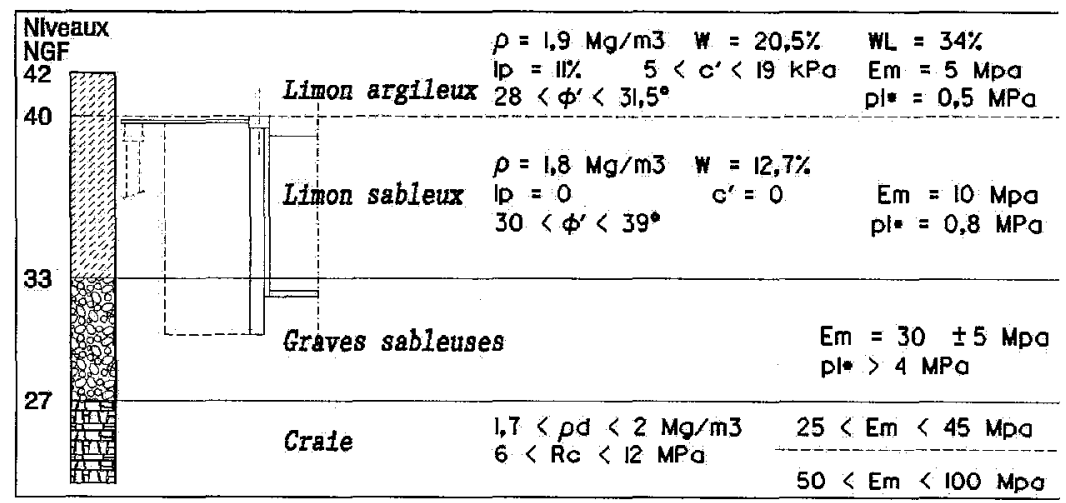

Figure 2 - Profil géotechnique au droit de la zone instrumentée et demi-coupe de l'ouvrage.

Figure 2-Geotechnical profile and tank cross section. 
La réalisation du bassin a débuté par le terrassement général d'une plate-forme à partir de laquelle ont été réalisés $1200 \mathrm{~m}$ de paroi moulée à contrefort constituant les bajoyers du bassin. Ces parois longitudinales sont constituées d'éléments de paroi moulée de 4,30 $\mathrm{m}$ de large et de $0,62 \mathrm{~m}$ d'épaisseur, comportant chacun un contrefort central de $3,88 \mathrm{~m}$ de long pour $0,62 \mathrm{~m}$ d'épaisseur (Figure 3a). Les parois moulées ont été recépées sur une hauteur de $1 \mathrm{~m}$ environ après préexcavation du bassin. L'excavation a ensuite été réalisée, en trois étapes, pour une profondeur totale de $8 \mathrm{~m}$, la fiche de la paroi restant au minimum égale à $1,40 \mathrm{~m}$. Des contre-voiles de $25 \mathrm{~cm}$ d'épaisseur nominale ont alors été coffrés le long des parois moulées afin d'obtenir les tolérances dimensionnelles et l'état de surface demandés pour les parements. Pour finir, le radier et les poutres de couronnement qui supporteront les rails pour la plate-forme de traction ont été réalisés (Figure 3b).

a)

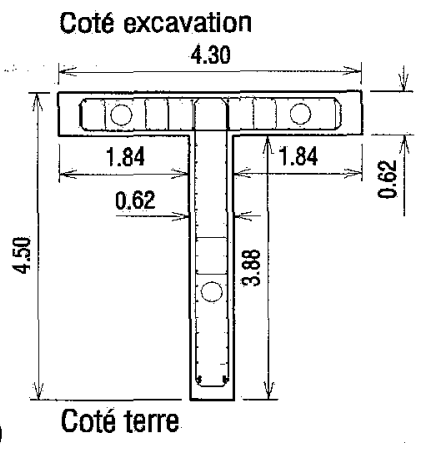

b)

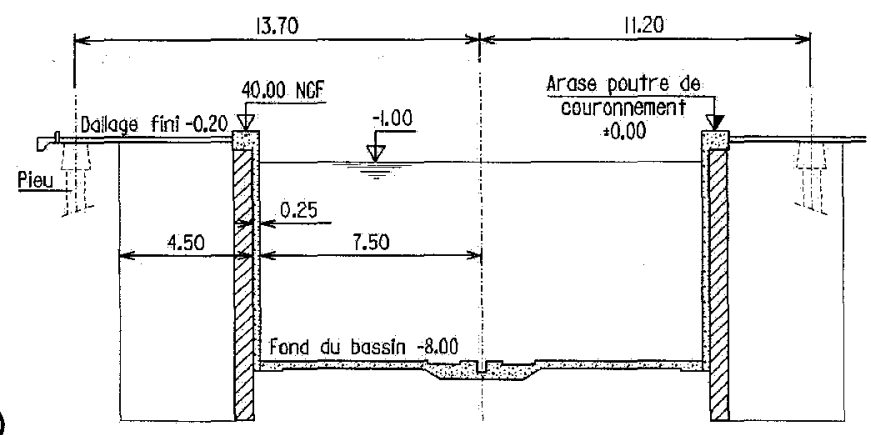

Figure 3 - Coupe horizontale d'un élément de paroi moulée (a) et vue en coupe du bassin (b). Figure 3 - Horizontal section of the diaphragm wall (a) and cross section of the tank (b).

\section{L'instrumentation et sa mise en auvre}

L'instrumentation (Figure 4) vise, d'une part, à rendre compte de la cinématique générale de l'ouvrage et du sol qui lui est associé et, d'autre part, à mesurer la déformation des contreforts et du voile de soutènement constituant la paroi. Deux profils sont instrumentés de façon à disposer d'une redondance minimale.

Le déplacement de la paroi est suivi par visée optique de sept plots topographiques. Ce suivi est complété par la mesure par fil invar de la convergence des deux parois en vis-à-vis. Cette dernière mesure intéresse des plots scellés dans la partie dégagée de la paroi au fur et à mesure de l'excavation et il s'agit donc d'une mesure de la convergence relative entre deux phases d'excavation et non d'une mesure de la convergence absolue. L'inclinaison de la paroi est mesurée au moyen de nivelles par le relevé de l'inclinaison de sept embases scellées dans la paroi. La déformée latérale du terrain est mesurée au moyen de tubes inclinométriques. La section principale de mesure est équipée de trois tubes de mesure inclinométrique de façon à disposer d'un profil complet de la déformation latérale du terrain en arrière de la paroi tandis que la section de 
contrôle est équipée de deux tubes inclinométriques seulement. Le suivi par visées optiques du déplacement, en plan et en altitude, de points situés à proximité des têtes des tubes inclinométriques permet de contrôler la qualité de l'intégration qui est faite lors de l'exploitation de la déformée des tubes inclinométriques et de compléter le profil de déformation latérale du sol par un profil de déplacement vertical de la surface du terrain (tassement de la surface du sol).

La déformation de la paroi moulée est mesurée au moyen d'extensomètres à cordes vibrantes et à jauges. Ces extensomètres sont répartis horizontalement et verticalement en différents niveaux du contrefort et du voile du profil principal d'instrumentation de l'ouvrage (Figure 4c et d).

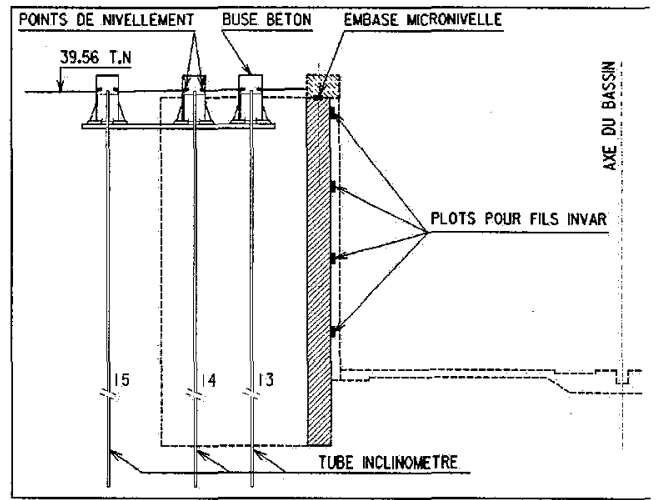

a)

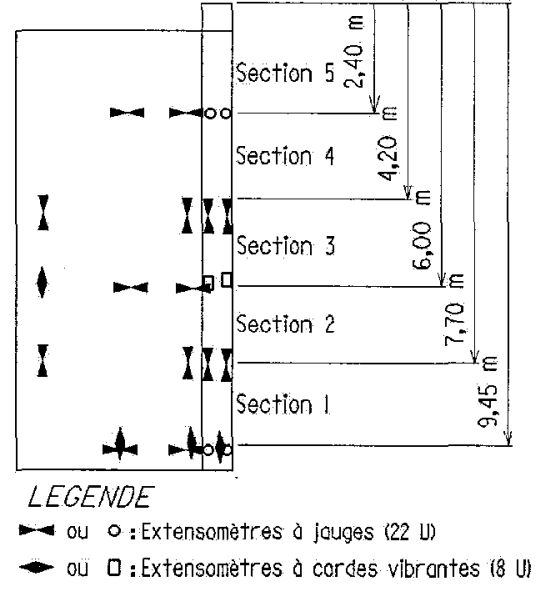

c)

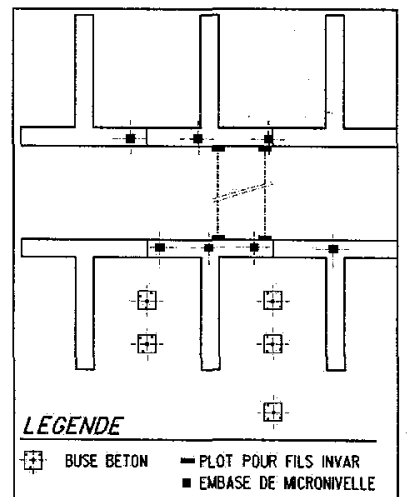

b)

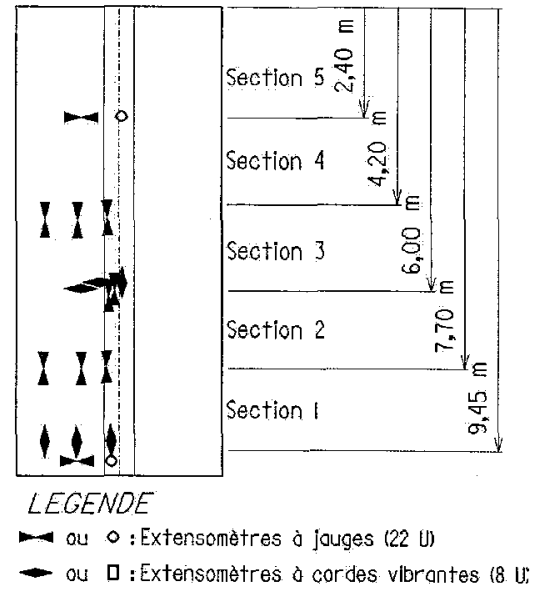

d)

Figure 4-Schéma de l'instrumentation : vues de côté (a) et de dessus (b) des inclinomètres et des plots de suivi du mouvement; vues de côté (c) et de face (d) des extensomètres.

Figure 4-Monitoring plan: side (a) and top (b) views of the position of inclinometers and surveying plots ; side (c) and front (d) views of the position of extensometers.

Les mesures sont effectuées de façon à rendre compte du comportement de l'ouvrage, d'une part, lors des phases les plus significatives de sa réalisation et de sa mise en service et, d'autre part, à plus long terme, en service. 


\section{Principaux résultats et perspectives}

La cinématique de l'ouvrage est très bien caractérisée par les relevés de mesures topographiques (Figure 5) et inclinométriques (Figure 6). Après des déplacements sensiblement égaux à zéro lors de la première phase d'excavation $(3 \mathrm{~m}$ de profondeur), l'excavation à $6 \mathrm{~m}$ de profondeur provoque un déversement de la paroi. Ce déversement prend naissance au-dessous du pied de la paroi, au niveau du toit de la couche de craie, le déplacement atteint en tête étant voisin de $3 \mathrm{~mm}$. Il est accompagné d'un mouvement latéral analogue $\mathrm{du}$ sol soutenu. L'amortissement de ce déversement avec la distance à la paroi est faible, les déplacements à la surface $d u$ sol restant tout juste inférieurs à $3 \mathrm{~mm}$ pour l'inclinomètre situé le plus en arrière. Ce déplacement latéral du sol soutenu s'accompagne d'un tassement de sa surface, d'amplitude maximale égale à $1 \mathrm{~mm}$ en arrrière immédiat de la paroi et décroissant avec la distance à la paroi. L'excavation à $8 \mathrm{~m}$ s'accompagne d'une cinématique différente, dont la composante principale est constituée d'une translation. Ce mouvement de translation intéresse la paroi sur sa hauteur et son intensité est comprise entre 1 et $2 \mathrm{~mm}$. Sous le pied de la paroi, ce déplacement latéral décroît pour s'annuler au niveau du toit de la couche de craie. Ce mouvement de la paroi est accompagné d'un mouvement analogue du sol soutenu, tant dans le profil, traduisant une translation, que dans l'amplitude, comprise entre 1 et $2 \mathrm{~mm}$. Le tassement du sol soutenu reste maximal en arrière immédiat de la paroi, de l'ordre d'une fraction de millimètre pour décroître avec la distance à la paroi.

Les déplacements latéraux de l'ouvage sont, en valeur cumulée, de l'ordre de 0,1 $\%$ de la hauteur d'excavation. Ils se situent donc dans la partie basse de la fourchette de déplacement donnée dans la littérature pour les soutènements d'excavations (Clough et O'Rourke, 1990).
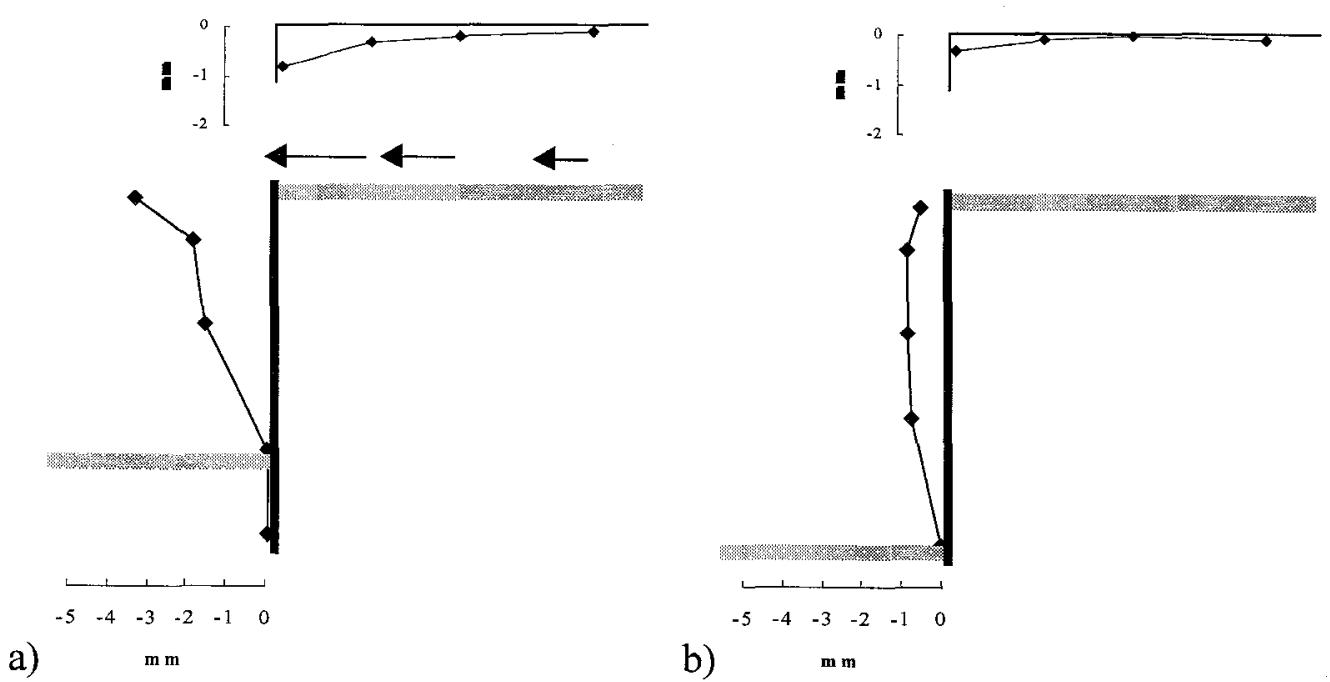

Figure 5 - Déplacement de la paroi et de la surface du sol : terrassement à $6 \mathrm{~m}$ (a) et à $8 \mathrm{~m}$ (b). Figure 5 -Horizontal movement of the wall and ground settlement : $6 \mathrm{~m}$ (a) and $8 \mathrm{~m}$ (b) deep excavation. 
a)

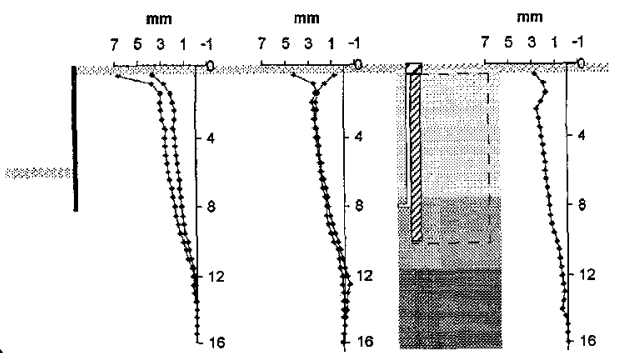

b)

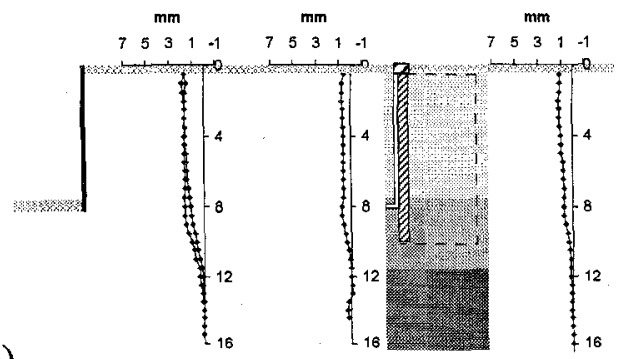

Figure 6 - Déplacement du terrain soutenu induit par l'excavation du bassin à $6 \mathrm{~m}$ (a) et à $8 \mathrm{~m}$ (b). Figure 7-Horizontal movement of supported ground: $6 \mathrm{~m}$ (a) and $8 \mathrm{~m}$ (b) deep excavation.

La déformation propre des éléments de paroi moulée est liće aux variations thermiques et aux actions mécaniques auxquelles elle est soumise. Les mesures de température, réalisées au moyen des sondes intégrées aux extensomètres, montrent que la prise du béton s'accompagne d'une élévation de sa température d'environ $15^{\circ} \mathrm{C}$, dont la dissipation dure environ deux mois. Cette évolution de la température s'accompagne d'un phénomène de retrait thermique auquel ont été sensibles les mesures de déformation en dépit de la compensation thermique des extensomètres. Les déformations induites dans la paroi moulée par le terrassement $\mathrm{du}$ bassin sont de faible amplitude, les valeurs maximales observées pour chacune des phases de terrassement valant de 10 à $20 \mu \mathrm{m} / \mathrm{m}$ (Figure 8), soit d'un ordre de grandeur voisin de celui des déformations induites par les variations de la température de la paroi. Il n'a en conséquence pas été possible de dissocier avec précision les déformations d'origine thermique des déformations d'origine mécanique et d'en déduire le schéma d'efforts induit dans la paroi moulée par l'excavation. Il est néanmoins possible de conclure à la faible mobilisation de la structure par rapport à sa capacité résistante.

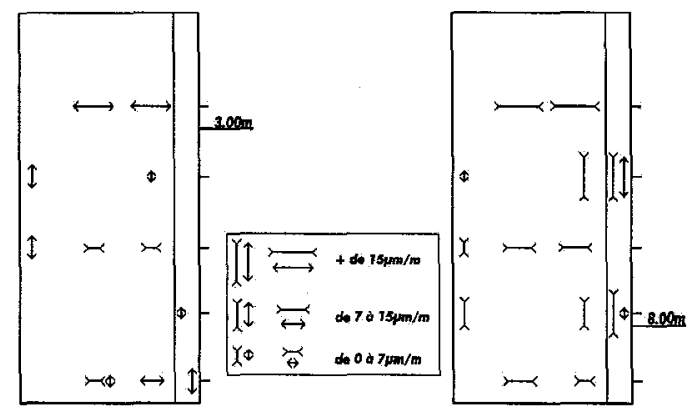

Figure 8 - Déformations du contrefort induites par le terrassement à $3 \mathrm{~m}$ et à $8 \mathrm{~m}$ du bassin. Figure 7-Measured strains of the diaphragm wall afterexcavation to $3 \mathrm{~m}$ and $8 \mathrm{~m}$ deep.

Un calcul rapide avec une approche au coefficient de réaction montre qu'un rideau sans contrefort dans le profil de terrain concerné devient instable pour une hauteur critique de terrassement de 6,7 m environ. Le déplacement en tête est alors de 17 $\mathrm{mm}$. Avec la mise en place des contreforts, la rigidité de la paroi se trouve augmentée, le centre de gravité de l'ensemble est reporté en arrière et des frottements sol/contreforts sont mobilisés. Cela conduit à assurer la stabilité de 
l'ouvrage et à minimiser ses déplacements. Le comportement de l'ouvrage se rapproche du comportement d'un ouvrage poids. Au stade actuel des analyses réalisées, il reste néanmoins difficile de statuer sur la nature précise de la mobilisation du massif de sol situé entre les contreforts. La mise en œuvre de simulations numériques, par la méthode des éléments finis, devrait permettre de répondre à cette question. Ce travail reste encore à réaliser.

\section{Conclusions}

L'étude expérimentale réalisée a permis de recueillir des données sur le comportement d'une paroi à contreforts. La cinématique de la paroi et du terrain emprisonné par les contreforts montre que leurs déplacements sont associés. Ces déplacements restent de faible amplitude conformément au comportement attendu et leur ordre de grandeur reste inférieur à celui des déplacements en général observés pour les soutènements d'excavations. La structure en béton armé des contreforts est très faiblement sollicitée au regard de sa capacité résistante. L'étude montre que le comportement de ce type de structure diffère sensiblement de celui d'une paroi plane pour se rapprocher de celui des ouvrages poids. Des travaux complémentaires, basés sur une simulation numérique, sont cependant nécessaires pour conclure sur la nature de la mobilisation du massif de sol situé entre les contreforts. Cette démarche devrait permettre de mieux comprendre l'importance relative de la longueur des contreforts et de leur espacement.

\section{Remerciements}

L'étude réalisée a associé les services de la DGA et de la Marine Nationale (STTIM) impliqués dans le projet et le réseau des Laboratoires des Ponts et Chaussées (LCPC, LRPC et CER de Rouen).

\section{Bibliographie}

Boloré, J., Cordier, S., 1999, Les nouveaux moyens d'essais en hydrodynamique, Revue Scientifique et Technique de la Défense, 43, pp. 9-19.

Cota, A., 1996, Le B600, un bassin pour le futur, Carènes, 6, pp. 6-9.

Clough, G. W., O'Rourke, T. D. (1990) - Construction induced movements of in situ walls. Proc. of the Conf. on Design and Performance of Earth Retaining Structures, Ithaca (New York) : Cornell University, pp. 439-470.

Delahaye, E., Haïun, G., Millan, A., 1998, Les ouvrages de soutènement-Guide de conception générale, Bagneux : SETRA, $154 \mathrm{p}$.

Lavisse, J., 1993, La paroi moulée autostable de la bibliothèque de France, Travaux, 689, pp. 42-49.

Parkinson, J., Guillaud, M., 1994, Conception, réalisation et suivi du comportement d'une paroi moulée autostable, Travaux, 697, pp. 23-29. 